\title{
Hierarchical Structure of Active Distribution @ Network in Power System
}

\author{
Desh Deepk Sharma, Atul Sarolwal
}

\begin{abstract}
The active distribution network (ADN) is an integral component of the smart grid. The ADN improves reliability and resiliency in the power grid integrated with many distributed energy resources (DERs). This is possible that, during outage, the $A D N$ can be isolated from the main grid and it can continue to operate in island mode with indeterminate broken links and scarce generation resources. With the active management of increasing DERs, the distribution network is changed to active distribution network from passive network. This paper reviews the characteristics and challenges of deployment of distributed power plants (DPPs) in hierarchical active distribution network

Keywords: Active distribution network, Distributed Power Plants, Distributed Energy Resources, Hierarchical Control, Competitive Control
\end{abstract}

\section{INTRODUCTION}

The active power distribution network can facilitate such automatic operations with high level of coordination and appropriate communication infrastructure. The big challenge for realization of active power distribution network is to adopt appropriate distributed control schemes. The system operator must be quick enough to detect the problems of DPSs and then respond. The improved power convertor technology enhances the control capabilities of distributed generation. In the active distribution network, there are hierarchies of multiple levels and interconnections with numerous distributed power units. In the active distribution network, the distributed power units have characteristics of scalability, modularity, flexibility, adaptability and autonomy. The smaller distributed power plants comprise solar power and storage devices, and these smaller plants can supply power locally and sell surplus power to the interconnected units. This poses the challenge to design the suitable information and control structures which can tackle both the technical and open market problems. These structures aggregate the information of services at local points in the system. An active power distribution network comprising various generators, storage devices and loads can represent a single power unit which is capable to be

Revised Manuscript Received on September 09, 2020

* Correspondence Author

Desh Deepak Sharma*, Associated Professor, Department of Electrical Engineering, MJP Rohilkhand University Bareilly, India. Email: deshdeepak101@gmai.com.

Atul Sarojwal, Assistant Professor, Department of Electrical Engineering, MJP Rohilkhand University, India, as@mjpru.ac.in

(C) The Authors. Published by Blue Eyes Intelligence Engineering and Sciences Publication (BEIESP). This is an open access article under the CC BY-NC-ND license (http://creativecommons.org/licenses/by-nc-nd/4.0/) dispatched like a controllable generation or loads during disturbances. The active power distribution network comprises of infrastructures of two tightly coupled networks of power and communication, and both networks have different distributed functionality [1]. With 'stand-alone' and 'plug-and-produce' functionalities, the distributed power systems (DPSs) are regarded as subsystems of the whole power system in which transmission and distribution networks are the different entities. In the DPSs, there are power exchanges with distributed power generations and other neighboring DPSs fig.1. In these controllable power units, the appropriate control and management schemes are applied such that effects of faults should not be propagated to other DPSs. Hence, reliability and resiliency of the system are improved with the integration of distributed power systems. With 'plug and produce' operation, the small distributed power units can frequently be connected and disconnected. With the increased penetration of distributed generations, the voltage profile of distribution networks is affected. A power factor control -voltage control method provides the efficient voltage regulation strategy on increased penetration of DG. In a decentralized control of distributed generation, the generator's profile is controlled in two modes as power factor control and voltage control at the point of connection to improve overall network connection [2]. The big challenge in the ADN is to develop coordination in different sources and loads. This ADN is characterized by uncertainty and stochasticity of output of distributed generators, and low inertia. This ADN must coordinate non-dispatchable distributed generation (photovoltaic and wind power), dispatchable distributed generation (synchronization motors and energy storage devices) and flexible loads [3]. This ADN possesses highly dynamic behavior and adopts distributed cooperative control methods. In cooperative control scheme, there is tight communication among network controllers. Each networked controller communicates with neighbor controllers. The uncertainty present in the $\mathrm{ADN}$, causes a sharp fluctuation of feeder power and voltage violation which result in more voltage and VAR control problems in the system and, hence, operating losses are increased, and stability issues are created in ADN [4]. The distribution network must be capable to adapt in tune with the variable generation obtained from different DERs. A distributed and multi-layered framework of ADN consists of three layers as first layer control, second layer and third layer. The first layer, known as global layer also,

Published By:

Blue Eyes Intelligence Engineering

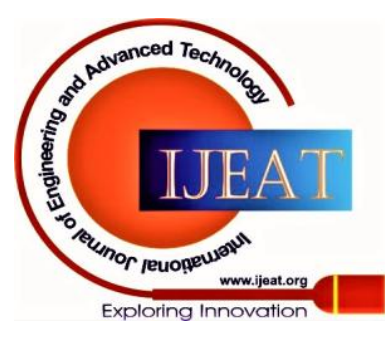




\section{Hierarchical Structure of Active Distribution Network in Power System}

possesses the global management system which sets the optimization goals for the lower layers with information of operational data of those layers. In the second layer, there are multiple sub-regions which, independently, maintain their own stability with autonomous control scheme. In the third layer, local devices such as DERs are controlled on the same distribution nodes, and thus fast response speed is achieved in the local control actions. The regional autonomous strategies such as constant feeder exchange power mode, regional autonomous control mode and regional cooperative control mode are generated by the indicator of feeder control error [5]. Let a radial distribution network is connected to the main grid and this distribution network is assumed a virtual power plant. A utilization ratio of each distributed generation is defined as the ratio of its active power to its maximal available power. A cooperative control strategy regulates the active power of the virtual power plant and a consensus is developed to maintain the utilization level each distributed generation (DG). A distributed confidence manager maintains the confidence level at each DG for all its in-neighbors [6]. A combined local and centralized control scheme is designed for voltage control of medium-voltage distribution networks by distributed generation units. The centralized upper layer aims to achieve coordination of various distributed generating units while model predictive control scheme is in the base of controller to adjust the VQ characteristics of local controllers. At lower level, fast response to disturbances are achieved with the local controller [7]. The ADN faces the challenge of voltage violations due to increased penetration of distributed generation. A soft point, a flexible power electronic device, is the continuous source of reactive power. A coordinated voltage and VAR control method considers the operation of soft point and multiple regulation devices to minimize operation costs and eliminate the voltage violations of ADN. This method for voltage and VAR control of large scale ADNs is efficient, provides global optimal solution, and has moderate computational burden. In this method, system operation costs comprise cost of power losses and switching operation, and constraints of system operator, on-load tap changer, switchable capacitor banks and tie switches are taken care [8]. A cyber physical system comprises the physical and cyber layers to connect the physical world to cyber layer. The cyber physical fusion modeling is being done with hybrid system model which can describe object by its dynamic rule, logical rule, operating constraints and a framework combining discrete and continuous features. The feeder control error changes if there is change in the power of intermittent energy resources and/or load demand. The autonomous control of an area aims to achieve feeder control error close to zero by controlling the set points for distributed generations of the same area [9]. The ADN can be decomposed into several areas and each area is equipped with a battery energy storage system (BESS). A real-time decentralized control algorithm based on BESS for multi-area active distribution network is designed. The area internal states are not shared with others and, thus, mutual dependency of the dynamics on that of others is reduced. The distributed control problem is implemented within an area while relying on the dynamics of BESS. Generally, there is radial structure in the distribution network so there is only one connection line between any two adjacent areas and common connection line between any two adjacent areas is known as common boundary bus for both areas. The partitioning of the distribution network into different areas is done on the following cases (a) changes in the topology of the network (b) connection or disconnection of a BESS [10]. Deployment of energy storage system at appropriate nodes of distribution network can solve the problems of overvoltage and undervoltage in low voltage feeders. With very limited information, the voltage problems are predicted, and storage control schemes are decided in advance to the voltage problems [11]. In the ADN, the multiple planning alternatives are reinforcement of existing substations and distribution lines, network expansions, active management of distributed generation with capacitor and voltage regulator placement. The multistage coordinated planning method optimizes the multiple planning alternatives for $\mathrm{ADN}$.An optimal operation of distribution network with joint active and reactive electricity market model is designed at distribution level with active distribution network (ANM) schemes integrated with demand response. In this design, the uncertainties associated with load demand and power generated by DERs are modelled [12]. Based on technical characteristics and economical operations of DGs, storage units, flexible loads, an agent based model of ADN is established for its optimal operation using price incentive information [13]. Coordinated operation of distributed generation, energy storage units and controllable loads is suggested in multi stage DNP planning with selection strategy for restricted operation scenarios based on the shadow price. In conventional Distribution Network Planning, operation cost is minimized considering network investment cost and some scenarios given by experts. In Active Distribution Network Planning, the difference between the actual scenarios and expected scenarios is considered. However, the considered scenarios are selected by impact of investment instead of by expertise [14].

This paper identifies the various challenges in design of hierarchical structure of active distribution network. In this paper, section 2 reviews the hierarchical control structure of distributed power plant, section 3 overviews the multi- layer coordination control of $\mathrm{ADN}$, section 4 discusses competitive power sharing control of $\mathrm{ADN}$, congestion management and voltage control are overviewed in section 5, in section 6 optimization mathematical models are discussed, section 7 identifies key challenges and then section 8 concludes.

\section{HIERARCHICAL CONTROL STRUCTURE OF DISTRIBUTED POWER PLANTS}

In active distribution network, there is inherent hierarchy at the different multi -levels and interconnections. So, hierarchical architecture must be explored to be implemented in the $\mathrm{ADN}$ for control and management purposes. The hierarchical distributed control scheme for distributed power plants, penetrated with numerous distributed generations and storage devices,

Published By:

Blue Eyes Intelligence Engineering and Sciences Publication

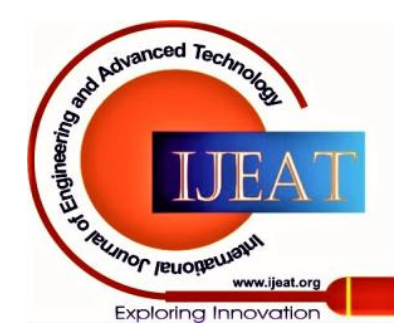


includes all functional levels from the instantaneous supervisory control of the resources to the long-term economic scheduling layer. This hierarchical control scheme focuses on all functionalities needed in operation and control of active power distribution plants.

The hierarchical control scheme facilitates the distributed power plants and responsive loads to participate in the retail markets such that maximum economic benefits over end-user power generation and demand needs can be achieved.

\section{A. Hierarchy in Control level}

The identified hierarchical control levels are enterprise level, area level, island level, power plant level, cluster level, station level and resource level. The enterprise level, a pure management unit, decides the economic long-term power schedule based on the participating generation units and the users using the power capacity forecasts. This level facilitates the participation of market players and system operators for finding the optimum trade of energy and grid services. The area level control, which is basically a distribution-sub transmission network, is capable to control secondary frequency and voltage using the real-time information of area level and perform in stand-alone mode from main grid whenever required. At this level, numerous distributed power plants are connected, and in a control area, several power plants are optimally managed within the conditions of DSO, fig.1. The DSO ensures safe and reliable operation of distributed systems with provision of advanced grid support services from distributed generation/demand units at area and island levels. At the power plant level, the power plant production is controlled on-site to cater the production schedule obtained from higher control levels. At cluster level, based on similar dynamics, the individual generating units are grouped in the clusters to implement flexible and coordinated control schemes. At this level, the affected cluster can be isolated during abnormal conditions. At station level, the generating units are locally controlled to deliver specified AC active and reactive power to the grid through power converters and synchronous generators. With energy extraction mechanism, active power supply from given resource generation unit is controlled. This hierarchical control methodology is general in structure of any sort of distributed power plant with the difference of final resource control method. In case of buildings or campuses, the building or campus energy management system hierarchical control structure replaces the power plant, cluster, station and resource levels [13]. In distributed network, distributed agents are considered for distributed generators in a distributed sparse network through hierarchical control method. The distributed hierarchical cooperative method performs cooperative optimization operation of source loads and maximization of economic benefits [14].

\section{B. Information flows}

In the distributed power system, the control unit hardware includes computer/control units (CU) and communication networks $(\mathrm{CN})$ at several control levels. The hierarchical structure of distributed control consists of field, process, unit, production and management. The specific data protocols are used for communication between control or computer units. The information flows through different levels, be executed and interact with other functional blocks in different control levels. At each control level, the information flows, be executed and used in different functionalities of different levels. The specific data protocols are used in communication between control units [1].

\section{MULTI-LAYER COORDINATION CONTROL IN} ADN

Despite several advantages of integration of DERs in the distribution grid, there are some challenges as well. The PI (proportional integration) control scheme is the basis for the design of existing control technologies for DERSs such as PQ control, PV control and Droop control. This control scheme is based on fit and forgets passive management and may not be an appropriate solution for control schemes to be implemented in ADN. The multi-layer control strategy required to be realized in the ADN which consists of different layers. In the ADN, the upper layer is active distribution management system (ADMS), in the middle layer there are different areas, and, in each area, there are various DERs and storage devices. The ADMS is built with central controller or supervisory controller, in the middle layer, there are layered distributed coordinated controllers, and, in the bottom layer, there are network coordinator controller. Using the information of practical operating state at different layers of $\mathrm{ADN}$, the different areas conduct coordination control scheme such that whole objective of $\mathrm{ADN}$ is optimized globally. If there is local change in the output of DER and/or electricity demand, the coordinated controllers adjust the output of battery energy storage systems (BESSs) or DERs such that power balance between local area and feeder can be achieved [14]. The coordinate control strategy based on feeder control error (FCE) is applied in autonomous areas of active distribution network aiming to obtain global optimization. FCE is defined to indicate power fluctuations in different areas and as given below,

$$
P_{F C E, j}=\alpha_{j} \times \Delta P_{\text {feeder }}+\Delta P_{a r, j}
$$

where $P_{F C E}$ is FCE value of área $j, \alpha_{j}$ is power coordination value coefficient of área $j, \Delta P_{\text {feeder }}$ is the deviation between actual exchange power of feeder and the optimal target value, $\Delta P_{\text {feeder }}=P_{\text {feeder }}-P_{\text {feeder }}^{o}$ and $P_{\text {feeder }}$ is actual exchange power of feeder and $P_{\text {feeder }}^{o}$ is the optimal target value of $P_{\text {feeder }}$ $\Delta P_{\text {area }, j}=P_{\text {area }, j}-P_{\text {area }, j}^{o}$,

$P_{\text {area,j } j}$ is actual exchange power of área $j$ and $P_{a r e a, j}^{o}$ is optimal target value $P_{\text {area,j }}$. If $\mathrm{FCE}<0$ or FCE $>0$ the storage unit and DG try to control their output powers to maintain FCE close to 0 in order to operate grid at globally optimum level [25].

\section{A. Loop power flow controller}

A loop flow controller (LPC) facilitates separate feeders to be connected by a multi-terminal AC-DC-AC converter. In distribution networks, LPCs are generally known as soft normally open points (SNOPs). In LPC, there are numerous voltage source converters (VSC) which are connected via a common DC link to loop the network and achieve power flow on separate feeder lines.

Published By:

Blue Eyes Intelligence Engineering

and Sciences Publication

(c) Copyright: All rights reserved.

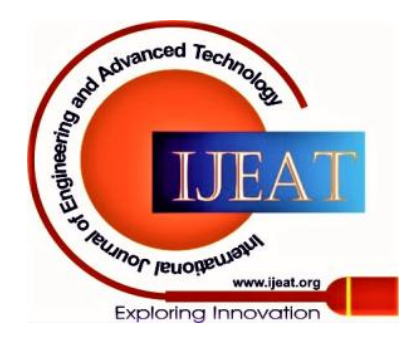




\section{Hierarchical Structure of Active Distribution Network in Power System}

In LPC-ADN, an example of the LPC with multiple terminals is connected to ADN and this LPC-ADN comprises a radial distribution network topology closed-looped by 4 LPCs including 3 two-terminal LPC and 1 three terminal LPC, 1 on-load tap changer (OLTC), 4 DGs and 2 capacitor banks. In LPC-ADN, without violating the limitations of short circuit currents, the power flows along different feeders can be flexibly exchanged and with different operational strategies of LPC the securities and efficiencies of LPC-ADN are improved. LPC is operated to balance the output of distributed generators by tracking their output fluctuations [19].

\section{VIRTUAL PRICE SIGNALS IN HIERARCHICAL STRUCTURE}

The competitive power control scheme aims to achieve real-time control of the plant generation such that there should be optimal allocation of available resources while minimizing operating costs. In this control scheme, final-end generation unit acts responsibly to decide its generation involvement. This involvement is decided using the information of its own marginal costs and virtual price signal which is received by it and propagated over entire hierarchical structure. The following price signals are generated in the hierarchical structure.

Station price signal $\left(\$_{\text {stat }}\right)$ - this price signal represents the price that each controller pays to the next controller (i.e. resource controller) connected to it. With this price signal, desired production objective at the convertor terminals is to be ensured. The resource controller compares this price with its own generation costs. The power production is decreased if the price signal is less than the resource costs and vice versa. Plant price signal $\left(\$_{\text {plant }}\right)$-The plant controller generates a real-time virtual price signal on the error between power plant reference power and power generated by it. This price signal is propagated to next level controller (cluster controller) where this price signal is modified. Plant price signal $\left(\$_{\text {plant }}\right)$-The plant controller generates a real-time virtual price signal on the error between power plant reference power and power generated by it. This price signal is propagated to next level controller (cluster controller) where this price signal is modified. Cluster Prices $\left(\$_{\text {clust }}\right)-$ This is the price each cluster will pay to each station controller connected to it on fulfilling the objective of generation at the cluster controller level. This price signal is further propagated to the station controllers connected to the same clusters. Based on operation costs of each individual station, modification to the cluster price signal is done. Station price signal $\left(\$_{\text {stat }}\right)$ - this price signal represents the price that each controller pays to the next controller (i.e. resource controller) connected to it. With this price signal, desired production objective at the convertor terminals is to be ensured. The resource controller compares this price with its own generation costs. The power production is decreased if the price signal is less than the resource costs and vice versa.

$$
\begin{gathered}
P_{\text {res }, k}=\int\left(\$_{\text {plant }}-C_{\text {plant }}-C_{\text {clust }, i}-C_{\text {stat }, j}\right. \\
\left.-C_{\text {res }, k}\right) d t
\end{gathered}
$$

where $C_{\text {plant }}$ be the plant-level related costs at plant level, $C_{\text {clust }, i}$ be cluster related cost of cluster $i$ at cluster level, $C_{s t a t, j}$ be the station level related cost of station $j$ at station level, and $C_{r e s, k}$ be resource level cost of resource $k$ at resource level. This equation is rewritten as

$$
\begin{aligned}
\frac{P_{\text {res }, k}}{d t}=\$_{\text {plant }}- & C_{\text {plant }}-C_{\text {clust }, i}-C_{\text {stat }, j} \\
& -C_{\text {res }, k}
\end{aligned}
$$

Through the energy distribution path, the intermediate system costs are used to decide the price signal generated by the plant controller, and, hence, the generation increment/decrement rate of final-end resource can be controlled. Thus, the cheaper resource is identified to contribute more in the plant production. In the competitive control scheme, all the controllers of the same hierarchical level receive the common price signal and try to achieve the desired production objective at the minimum cost. Therefore, the generation unit with lower overall marginal costs generates power earlier compared to the generation unit with high marginal costs. In the competitive control scheme, the generation resources are optimally allocated and the desired production objective at the minimum operative costs is fulfilled. At any control level, the price control signal is generated based on the information of received price signal from higher immediate level controller and own distribution system performance, and no information of associated lower level controller is needed. Thus significant amount of information exchanged and consequently processing are saved. At the distribution level, there are large penetration of DERs and energy storage devices, and the loads may be controllable or noncontrollable. Due to the large diversity at different levels, an appropriate hierarchical control scheme is to be developed for distributed power plants.

\section{CONGESTION MANAGEMENT AND VOLTAGE CONTROL}

The hosting capacity of network for distribution generation is limited by voltage rise issues in the weak distribution network. Also, the hosting capacity of network for load is limited by voltage drop issues. The traditional solutions for mitigating the voltage rise/drop are either increasing the conductor size or connection of generator on dedicated feeder, and active voltage control methods. In $\mathrm{ADN}$, the power flow control methods are implemented to avoid congestions in the network which generally occur due to overloading of transformer or line section. The dynamic incentive or non-incentive based dynamic grid tariffs can be adopted to shift load demand to off-peak hours. In active distribution network, flexibility services such as rescheduling of controllable loads, storage devices and DG units obtained from aggregator are possible to realize.

\section{A. Congestion Management}

The congestion can be managed in three hierarchical levels as primary, secondary and tertiary. 
The primary level does not possess total information of the whole system and, thus at this level, control schemes are designed based on local measurements. The possible control schemes are voltage control with transformer OLTC, reactive power regulation of compensators, reactive power regulation of DG units.

At the secondary control level, the states of MV or LV network are estimated, and coordinated control scheme is implemented for primary controllers and direct control of load shedding, energy storage usages or generation reduction within an area. Tertiary control, located at control centre, validates the states of the network with appropriate tool. At this level, the topology of whole network is identified, controllers are coordinated and flexible services from aggregator are implemented. The coordination of controllers enhances the hosting capacity of the active network.

\section{B. Voltage Control}

In [6], two levels of voltage control scheme are proposed for real time adjustment of abnormal voltages in active distribution network. At local level, if there is terminal variation then local controllers equipped in the distributed generation units (DGUs) adjusts the reactive power. At upper level, discrete time centralized controller receives inputs of voltage and reactive power measurement from local controllers and decides the set pints. It is assumed that the received measurement has no communication delay. At local level, using steady state VQ characteristics and generic model of DGU reactive power model, the desired reactive power is obtained as shown below.

$$
\left\{\begin{array}{cc}
Q_{\max } & \text { if } V-\Delta V \leq V_{\min 2}^{t h} \\
R\left(V_{\min 1}^{t h}+\Delta V-V\right) & \text { if } V_{\min 2}^{\text {th }}<V-\Delta V<V_{\min 1}^{\text {th }} \\
0 & \text { if } V_{\min 2}^{\text {th }}<V-\Delta V<V_{\max 1}^{t h} \\
-R\left(V-V_{\max 1}^{t h}-\Delta V\right) & \text { if } V_{\max 2}^{\text {th }}<V-\Delta V<V_{\max 1}^{\text {th }} \\
Q_{\min } & \text { if } V-\Delta V \geq V_{\max 2}^{t h}
\end{array}\right.
$$

where $V_{\min 1}^{t h}, V_{\min 2}^{t h}, V_{\max 1}^{t h}$ and $V_{\max 2}^{t h}$ are different voltage thresholds, $\Delta V$ is the voltage shift corresponding to the cumulated reactive power correction $\Delta C$ and given as $\Delta V=\Delta C / R, R$ is the local droop of the VQ characteristics. At centralized level, the deviation of the reactive power is formulated in multi-step model predictive control optimization.

$$
\begin{gathered}
\min _{Q_{g}, V, \epsilon, \Delta Q_{g}, \Delta Q_{c o r}} \sum_{i=0}^{N_{c}-1}\left\|\Delta Q_{g}(k+i)\right\|_{W_{Q}}^{2}+\|\in\|_{S}^{2} \\
\Delta Q_{g}(k+i)=Q_{g}(k+i)-Q_{g}^{m}(k)
\end{gathered}
$$

where $\left(i=0, \ldots \ldots, N_{c}-1\right), W_{Q}$ is a diagonal matrix allowing to give different weights to different DGUs. The $\epsilon$ is aimed at relaxing the inequality constraints in case of infeasibility. Matrix $S$ is also diagonal with large diagonal elements to force the constraints. The $L_{2}$ norm a large number of DGU among those that can help correcting the voltage problem.

\section{Coordinated Voltage Control}

In active distribution network, non- operating point (NOP) is being replaced by soft operating point (SOP) between neighboring feeders a power electronics device possessing better controllability. In ADN, SOP can efficiently isolate fault and restore supply. In the following objective function, operational cost and voltage deviation are minimized.

$\min Q=\omega_{L}\left(Q_{\text {loss }}+Q_{\text {switch }}\right)+\omega_{v} Q_{v}$

where $Q_{\text {loss }}$ be the cost of power losses, $Q_{\text {switch }}$ be the cost of switching, $Q_{v}$ decides to minimize the extent of voltage deviation from desired value, $\omega_{L}$ and $\omega_{v}$ are the weight coefficients. In ADN, network losses and power losses caused by the power transmission of SOP are contained in cost of power losses. The adjusting cost of on load tap changers (OLTC) and the switching cost of CBs make the overall switching cost [6].

In active distribution network, non- operating point (NOP) is being replaced by soft operating point (SOP) between neighboring feeders a power electronics device possessing better controllability. In ADN, SOP can efficiently isolate fault and restore supply.

In the following objective function, operational cost and voltage deviation are minimized.

$\min Q=\omega_{L}\left(Q_{\text {loss }}+Q_{\text {switch }}\right)+\omega_{v} Q_{v}$

where $Q_{\text {loss }}$ be the cost of power losses, $Q_{\text {switch }}$ be the cost of switching, $Q_{v}$ decides to minimize the extent of voltage deviation from desired value, $\omega_{L}$ and $\omega_{v}$ are the weight coefficients. In ADN, network losses and power losses caused by the power transmission of SOP are contained in cost of power losses. The adjusting cost of on load tap changers (OLTC) and the switching cost of CBs make the overall switching cost [6].

One of the challenge in active distribution network is to avoid voltage fluctuations at different node due to a large number of connection of various kind of distributed generations at different nodes. Voltage control and line congestion management are considered in the proposed area control scheme. The ADN is considered balanced, radial and to be comprised of several quasi-autonomous areas controlled by a distributed energy source system. The clustered real time adaptive model based control scheme is applied in each area such that each area shares active/reactive power limits [9]. The optimization in each area is shown below.

$$
\begin{array}{r}
\min _{P_{b e s s}^{a c}, Q_{b e s s}^{a c}, P_{c b b}^{a c}, Q_{c b b}^{a c}} \alpha_{1} f_{1}(t)+\alpha_{2} f_{2}(t) \\
+\alpha_{3} f_{3}(t)+\alpha_{4} f_{4}(t)
\end{array}
$$

where $\alpha_{1}, \alpha_{2}, \alpha_{3}$ and $\alpha_{4}$ are the weights of the objective function, $f_{1}, f_{2}, f_{3}, f_{4}$ are operation cost of battery energy storage system, area node voltage regulation, cost of required active power from neighbors, area line current flow regulation, respectively, $P_{\text {bess }}^{a c}, Q_{\text {bess }}^{a c}$ are AC active/reactive powers of battery energy storage system, respectively, , $P_{c b b}^{a c}, Q_{c b b}^{a c}$ are active reactive power at common boundary bus. All these powers are constrained by their maximum and minimum values.

\section{OPTIMIZATION MATHEMATICAL MODEL FOR AND}

Published By:

Blue Eyes Intelligence Engineering

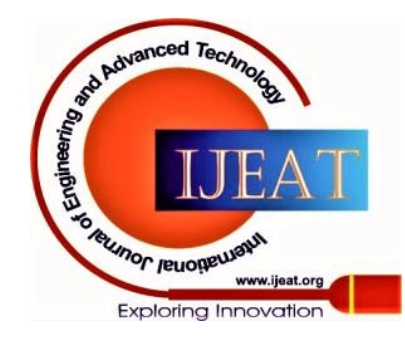




\section{Hierarchical Structure of Active Distribution Network in Power System}

\section{A. Hierarchical control cooperative optimization}

A multistage planning coordinated framework with different active network management (ANM) schemes is suggested for the active distribution network which comprises massive distributed generating units.

The active management of distribution network comprises coordinated control of both DG units and network components. The ANM schemes simultaneously considers reinforcement of the existing substation and distribution lines, the expansion, placement of circuit breakers and voltage regulators. The distribution is planned under maximum stress conditions. The power distribution network planning problem is formulated as MINLP problem as given below,

$$
\begin{aligned}
\min F=\sum_{t=1}^{T}\left(\frac{1+R_{\text {inf }}}{1+R_{\text {int }}}\right)^{t}\left(C I_{s r, t}+C I_{l r, t}\right. \\
\left.+C I_{l a, t}+C I_{c b, t}+C I_{v r, t}\right)
\end{aligned}
$$

where $R_{\text {inf }}$ is the inflation rate, $R_{\text {int }}$ is the interest rate, $C I_{s r, t}, C I_{l r, t}, C I_{l a, t}, C I_{c b, t}, C I_{v r, t}$ are cost of investment might occur in reinforcement of the substations, existing distribution lines, expansion of distribution network, placement of CBs, installation of VRs [11].

\section{B. Stochastic model predictive control}

For active distribution network, stochastic receding horizon control based on stochastic model predictive control is proposed for minimizing voltage deviation from pre decided set points and cost of controlling different controllable generating resources considering degradation cost of battery energy storage system and promoting low carbon electricity. The suggested objective function is as follows

$$
\begin{aligned}
& \min J \\
& =E_{x(t \mid t)}\left[Q_{1}(x)+Q_{2}(u)+Q_{3}\left(e^{s u}\right)\right. \\
& +Q_{4}\left(P^{C P}, Q^{C P}\right) \\
& \left.+Q_{5}\left(P^{P V}, \hat{P}^{P V}, P^{W T}, \hat{P}^{W T}\right)\right]
\end{aligned}
$$

where $Q_{1}(),. Q_{2}(),. Q_{3}(),. Q_{4}($.$) and Q_{5}($.$) refer to the$ cost of voltage deviations, control costs of various controllable resources, the degradation cost of storage units, the power exchange tariff to the utility and the cost penalizing renewable generation abandonment respectively. $e^{s u}$ represents the $x(t)$ be the voltage magnitude of all the buses (p.u.). $Q_{1}($.$) and Q_{2}($.$) are defined using the model$ predictive control scheme, degradation cost of storage units is defined using its number of remaining cycles, power exchange tariff is defined using prices of active and reactive power, curtailment cost is defined based on penalizing energy curtailment of PVs and WTs [24].

\section{KEY CHALLENGES}

Massive connection of DGs in different areas/levels create operational challenge in active distribution network. DGs power set points are difficult to set as these are not available for direct control by distribution network utilities, hence, real time control is a big challenge. The congestion management is the another challenge due to this massive connection of DGs, hence, network performance may degrade in the hierarchical network of ADN. The hierarchical network has high $\mathrm{R} / \mathrm{X}$ ratios, the reactive power compensation method might not suffice to control voltage. Such as, the reactive power injection deployed by DR regulator with droop control methods may not able to maintain voltage fluctuation within $+/-10 \%$. Complex ICT infrastructure is need to connect all entities and hardware for achieving better real time control and management. Coordination of different controllers used at different levels in the network is the big challenge for implementing real time optimal control scheme in the distributed system.

\section{CONCLUSION}

This paper reviews different features of the active distribution network which, generally, comprise hierarchical structure. In this hierarchical structure, different levels are discussed for control and management purposes. The coordinated control scheme has been identified in this multi-layer control system. The challenge of congestion management in $\mathrm{ADN}$ has been explored as given in different literatures. Also, the challenge of implementation of cooperative optimization algorithm has been surveyed. The idea of development of price based hierarchical control scheme for distributed power plants has also been identified.

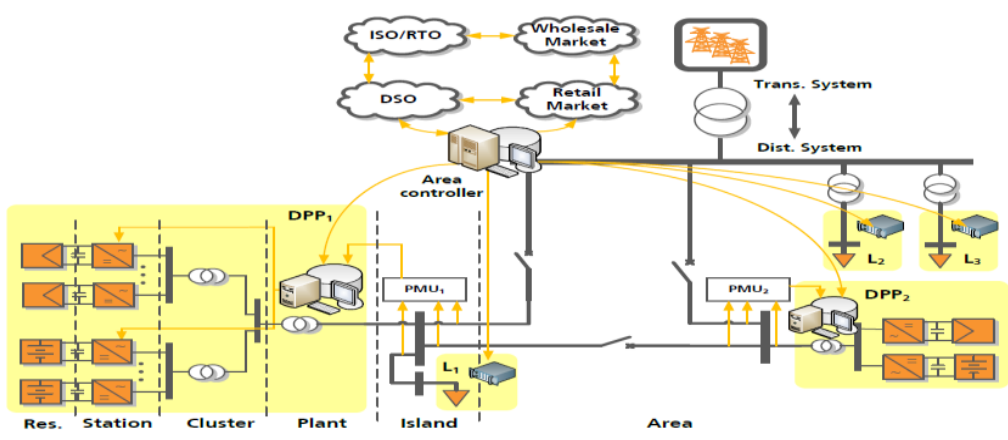

Fig. 1.Hierarchical network structure in active distribution grids[11]

Published By:

Blue Eyes Intelligence Engineering and Sciences Publication

(C) Copyright: All rights reserved.

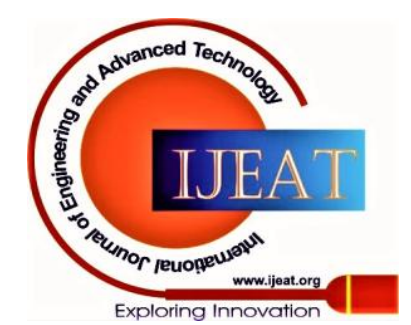




\section{REFERENCES}

1. J. Svensson, "Active Distributed Power Systems Functional Structures for Real-Time Operation of Sustainable Energy Systems," Department of Industrial Electrical Engineering and Automation, Lund Institute of Technology., Lund, 2006.

2. T. Sansawat, J. O'donnel, L. F. Ochoa and G. P. Harrison, "Decentralized voltage control for active distribution networks," in 2009 44th International Universities Power Engineering Conference (UPEC), Glasgow, UK, 2009.

3. L. Wu, L. Jiang, X. Hao and T. Zheng, "Research on distributed cooperartive optimization control strategy for active distribution network based on combine-then-adapt diffusion algorithm," The Journal of Engineering, vol. 2019, no. 16, pp. 1911-1917, 2018.

4. P. Li, H. Ji, C. Wang, J. Zhao, G. Song, F. Ding and J. Wu, "Coordinated control method of voltage and reactive power for active distribution networks based on soft open point," IEEE Transactions on Sustainable Energy, vol. 8, no. 4, pp. 1430-1442, 2017.

5. J. Zhou, D. Liu, B. Shen, J. Liu and C. Fang, "Active distribution network layered and distributed control strategy and implementation," in 2014 China International Conference on Electricity Distribution (CICED 2014), Shenzhen , 2014.

6. Y. Liu, H. Xin, Z. Qu and D. Gan, "An attack-resilient cooperative control strategy of multiple distributed generators in distribution networks," IEEE Transaction on Smart Grid, vol. 7, no. 6, pp. 2923-2932, 2016.

7. S. H. Bidgoli and T. V. Cutsem, "Combined local and centralized voltage control in active distribution networks," IEEE Transactions on power systems , vol. 33, no. 2, pp. 1374-1384, 2018.

8. P. Li, H. Ji, C. Wang, J. Zhao, G. Song, F. Ding and J. Wu, "Coordinated control method of voltage and reactive power for active distribution networks based on soft open point," IEEE Transactions on Sustainable Energy, vol. 8, no. 4, pp. 1430-1441, 2017.

9. M. Bahramipanah, D. Torregrossa, R. Cherkaoui and M. Paolone, "A decentralized adaptive model-based real-time control for active distribution networks using battery energy storage systems," IEEE Transactions on Smart Grid, vol. 9, no. 4, pp. 3406-3418, 2018.

10. D. Zarrilli, A. Giannitrapani, S. Paoletti and A. Vicino, "Energy storage operation for voltage control in distribution networks : A receding horizon approach," IEEE Transactions on Control Systems Technology, vol. 26, no. 2, pp. 599-609, 2018.

11. N. C. Koutsoukis and P. S. H. N. D. Georgilakis, "Multistage coordinated planning of active distribution networks," IEEE Transactions on Power Systems, vol. 33, no. 1, pp. 32-44, 2018.

12. A. M. Cantarellas, D. Remon, J. M. Garcia and P. Rodriguez, "Competitive control of wave power plants though price-signal optimum allocation of available resources," in IEEE Energy Conversion Congress and Exposition (ECCE), Cincinati, OH, USA , 2017.

13. R. H.A. Zubo, G. Mokryani, "Active distribution network operation: A market based approach" IEEE Systems Journal, vol. 1, no. 1, pp. 405-1416, 2020.

14. S. Hu, Y. Xiang, J. Liu, C. Gu, X. Zhang, Y. Tian, Z. Liu, J. Xiong, “ Agent-based coordinated operation strategy for active distribution network with distributed energy resources" IEEE Transactions on Industry Application, vol.55, no. 1, pp.3310-3320, 2019.

15. L. Zhao, Y. Huang, Q. Dai, L. Yang, F. Chen, L. Wang, K. Sun, J. Huang, A. Z. Lin "Multistage active distribution network planning with restricted operation scenario selection" IEEE Access, vol. 7, pp. 121067-121080, 2019

16. C. Li, S. Miao, Y. Li, D. Zhang, C. Ye, Z. Liu, L Li, “Coordinating dynamic network reconfiguration with ANM in active distribution network optimization considering system structure security evaluation" IET Generation, Transmission \& Distribution, vol. 13, no. 19, pp. 4355-4363, 2019.

17. L. Wu, L. Jaing, X. Hao and T. Zheng, "Research on distributed cooperative optimization control strategy for active distribution network based on combine-then-adapt diffusion algorithm," Journal of Engineering , vol. 2019, no. 16, pp. 1911-1917, 2019.

18. Y. Dexiang and L. Dong, "Application of model predictive control in active distribution network," in China International Conference on Electricity Distribution (CICED 2014), Shenzhen, 2014.

19. X. Xing, J. Lin, C. Wan and Y. Song, "Model predictive control of LPC-looped active distribution network with high penetration of distributed generation," IEEE Transactions on Sustainable Energy, vol. 8, no. 3, pp. 1051-1063, 2017.

20. P. Li, H. Ji, C. Wang, J. Zhao, C. Wang, J. Zhao, G. Song, J. Wu, "Coordinated control method of voltage and reactive power for active distribution networks based on soft open point" IEEE Transactions on Sustainable Energy, vol. 8, no. 4, pp. 1430-1442, 2017.

21. M. Bahramipanah, D. Torregrosa, R. Cherkaoui, M. Paolone, "A decentralized adaptive model- based real- time control for active distribution network using battery energy storage systems, IEEE Transactions on Smart Grid, vol. 9,no. 4, pp. 3406- 3418, July 2018.

22. A.S. Bouhouras, C. Iraklis, G. Evmiridis, and D. P. Labridis, "Mitigating distribution network congestion due to high DG penetration," in Proceedings $9^{\text {th }}$ Mediterrr exhibition Power Generation Transmission Distribution Energy Converters (Med Power), Athens, Greece, Nov, 2014.

23. N. C. Koutsoukis, P. S. Georgilakis, N. D. Hatziargyriou, "Multistage coordinated planning of active distribution network," vol. 33, no. 1, pp 32-44, 2018.

24. Y. Jiang, C. Wan , J. Wang, Y. Song, and Z. Y. Dong, "Stochastic receding horizon control of active distribution networks with distributed renewables" vol. 34, no. 2, pp. 1325-1341, 2019.

25. L. Wei, L. Dong, and D. Hui, 'Research of area coordinate control based on cyber-physical fusion modeling in active distribution network' in 2016 China International Conference on Electricity Distribution (CICED 2016), Xi'an.

\section{AUTHORS PROFILE}

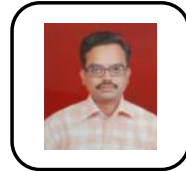

Dr. Desh Deepak Sharma(MIEEE) received B.E, M. Tech. and Ph. D. degree from MMM Engineering College Gorakhpur, NIT Kurukshetra, IIT Kanpur respectively. He was selected for post-doc and worked at Loyola Institute of Science and Technology, UNIVERSIDAD LOYOLA ANDALUCÍA, Seville, Spain during 2019. He worked on research project titled as "An Integrated Platform for In credited Flexibility in smart Transmission grids with STORAGE Entities and Large penetration of Renewable Energy Sources (FLEXITRANSTORE) (A European Union's Horizon 2020 research and innovation programmed under grant agreement No 774407). His research interest are demand side management, application of data mining techniques in load profiling and distribution system, distributed control schemes for distributed generation and energy storage systems, multi-agent system for power system, smart grid as cyber-physical system, and cyber security issues in power system, application of multi-agent system/Game theory in TSO/DSO based European Electricity market incorporating energy storage system and to develop new business models.

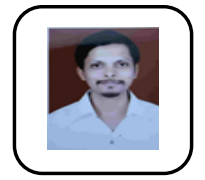

Dr. Atul Sarojwal, Working as Assistant Professor in the department of Electrical Engineering, Faculty of Engineering \& Technology, MJP Rohilkhand University, Bareilly since 2008. Also worked as Assistant Professor in SRMSCET Bareilly from 2005 to 2008. Total 15 years of teaching experience. Received Ph.D in Electrical Engineering from Invertis University, Bareilly in 2019. Patent of Ph.D research work is in progress. Completed B.Tech in the year 2003 and received Gold Medal in B.Tech course. Published 8 papers in international journals and conferences. Organized many national, international workshops and faculty development programs and also participated in many workshops and FDPs

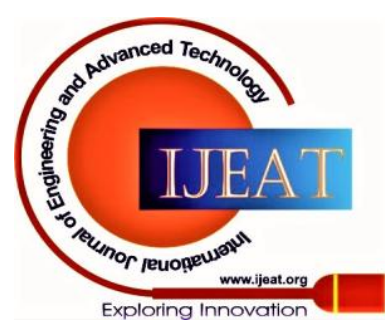

